\title{
Transport Properties of a 3D Topological Insulator based on a Strained High-Mobility HgTe Film
}

\author{
D. A. Kozlov, ${ }^{1,2,{ }^{*}}$ Z. D. Kvon, ${ }^{1,2}$ E. B. Olshanetsky, ${ }^{1}$ N. N. Mikhailov, ${ }^{1}$ S. A. Dvoretsky, ${ }^{1}$ and D. Weiss ${ }^{3}$ \\ ${ }^{1}$ A. V. Rzhanov Institute of Semiconductor Physics, Novosibirsk 630090, Russia \\ ${ }^{2}$ Novosibirsk State University, Novosibirsk 630090, Russia \\ ${ }^{3}$ Experimental and Applied Physics, University of Regensburg, D-93040 Regensburg, Germany
}

(Received 14 June 2013; published 14 May 2014)

\begin{abstract}
We investigate the magnetotransport properties of strained $80 \mathrm{~nm}$ thick $\mathrm{HgTe}$ layers featuring a high mobility of $\mu \sim 4 \times 10^{5} \mathrm{~cm}^{2} / \mathrm{V} \cdot \mathrm{s}$. By means of a top gate, the Fermi energy is tuned from the valence band through the Dirac-type surface states into the conduction band. Magnetotransport measurements allow us to disentangle the different contributions of conduction band electrons, holes, and Dirac electrons to the conductivity. The results are in line with previous claims that strained $\mathrm{HgTe}$ is a topological insulator with a bulk gap of $\approx 15 \mathrm{meV}$ and gapless surface states.
\end{abstract}

DOI: 10.1103/PhysRevLett.112.196801

PACS numbers: 73.25.+i, 05.60.Gg, 73.20.At, 73.43.-f

The discovery of two- (2D) and three-dimensional (3D) topological insulators (TIs), a new material class with insulating bulk and topologically protected conducting surface states, has opened an exciting research field in condensed matter physics [1-10]. Although quite a number of different, especially, Bi-based materials [11-14], belong to this category, materials which combine high charge carrier mobility and insulating bulk are still scarce. This is mostly due to the fact that Bi-based 3D TIs are heavily doped alloy films with a low mobility $\approx 1000 \mathrm{~cm}^{2} / \mathrm{V} \cdot \mathrm{s}$ and a high bulk carrier density of $10^{17}-10^{19} \mathrm{~cm}^{-3}$. HgTe-based 2D TIs, on the other hand, are characterized by very high mobilities enabling the discovery of the quantum spin Hall effect [15]. A recent analysis of the sequence of quantum Hall plateaus suggests that also strained HgTe layers constitute a 3D TI. The strain opens a gap in the gapless semimetal $\mathrm{HgTe}$ so that the TI properties can be explored by tuning the Fermi energy $E_{F}$ into the bulk gap and probing the transport properties of the gapless surface states. Although the strained HgTe film has a much higher mobility $\mu=(3-4) \times 10^{4} \mathrm{~cm}^{2} / \mathrm{V} \cdot \mathrm{s}$, the high bulk carrier density and the absence of a top gate have complicated the detection of 3D TIs so far $[16,17]$.

The strain in HgTe layers grown by molecular beam epitaxy stems from a $0.3 \%$ lattice mismatch between $\mathrm{HgTe}$ and CdTe. The corresponding critical film thickness is larger than $100 \mathrm{~nm}$, meaning that thinner films adopt the substrate lattice constant. Because of this strain, a small gap of $\sim 15 \mathrm{meV}$ opens (see below) in the bulk energy spectrum of the film. Within the bulk gap, the gapless surface states reside. The charge neutrality point of the corresponding Dirac cone is located in the valence band [16].

In this Letter, we report on transport properties of highmobility, $80 \mathrm{~nm}$ wide, strained $\mathrm{HgTe}$ films equipped with a gate. The low disorder manifested in high charge carrier mobilities, together with the possibility to tune $E_{F}$ from the valence via the gap into the conduction band, enables us to probe the 2D Dirac surface states when $E_{F}$ is in the bulk energy gap. Since HgTe films grown on CdTe suffer from dislocations due to the lattice mismatch, our $80 \mathrm{~nm}$ thick HgTe films were separated from the CdTe substrate by a $20 \mathrm{~nm}$ thin $\mathrm{Cd}_{0.7} \mathrm{Hg}_{0.3} \mathrm{Te}$ buffer layer. This buffer layer increases the electron mobility by an order of magnitude (up to $4 \times 10^{5} \mathrm{~cm}^{2} / \mathrm{V} \cdot \mathrm{s}$ ) and reduces the bulk impurity concentration to values of order $10^{16} \mathrm{~cm}^{-3}$ (see the Supplemental Material [18]). We fabricated and investigated two types of devices: one with the upper $\mathrm{HgTe}$ surface uncapped and the other one covered with a $20 \mathrm{~nm}$ $\mathrm{Cd}_{0.7} \mathrm{Hg}_{0.3}$ Te cap layer (see Fig. 1(a) and the Supplemental Material [18]). Since all measured major properties (magnetotransport traces, bulk energy gap, etc.) of capped and uncapped films were similar, we will only focus on the uncapped $\mathrm{HgTe}$ film below. For transport measurements, the films were patterned into Hall bars (see the Supplemental Material [18]) supplied with top gates. Cross sections of the devices are sketched in Fig. 1(a). For gating, two types of dielectric layers were used, giving similar results: $100 \mathrm{~nm} \mathrm{SiO} 2$ and $200 \mathrm{~nm}_{2}$ of $\mathrm{Si}_{3} \mathrm{~N}_{4}$ grown by chemical vapor deposition or $80 \mathrm{~nm} \mathrm{Al}_{2} \mathrm{O}_{3}$ grown by atomic layer deposition. In both cases, TiAu was deposited as a metallic gate. Magnetotransport measurements were performed at temperatures $T$ between 1.5 and $15 \mathrm{~K}$ and in magnetic fields $B$ up to $10 \mathrm{~T}$. Several devices from the same wafer have been studied.

Figure 1(b) shows the typical resistivity $\rho_{x x}$ at $B=0$ and Hall resistance $\rho_{x y}$ at $B=1 \mathrm{~T}$ as a function of gate voltage $V_{g}$ at $T=1.9 \mathrm{~K}$ for a $\mathrm{HgTe}$ film with a $\mathrm{Si}_{3} \mathrm{~N}_{4}$ insulator. The $\rho_{x x}$ trace exhibits a maximum near $V_{g}=1 \mathrm{~V}$ and is asymmetric with respect to $V_{g}$ : the resistance on the lefthand side of the maximum is significantly higher than on the right side. While $\rho_{x x}$ displays a maximum in Fig. 1(b), $\rho_{x y}$ taken at $1 \mathrm{~T}$ changes sign at the same $V_{g} \sim 1 \mathrm{~V}$. This 
(a)

TiAu Gate
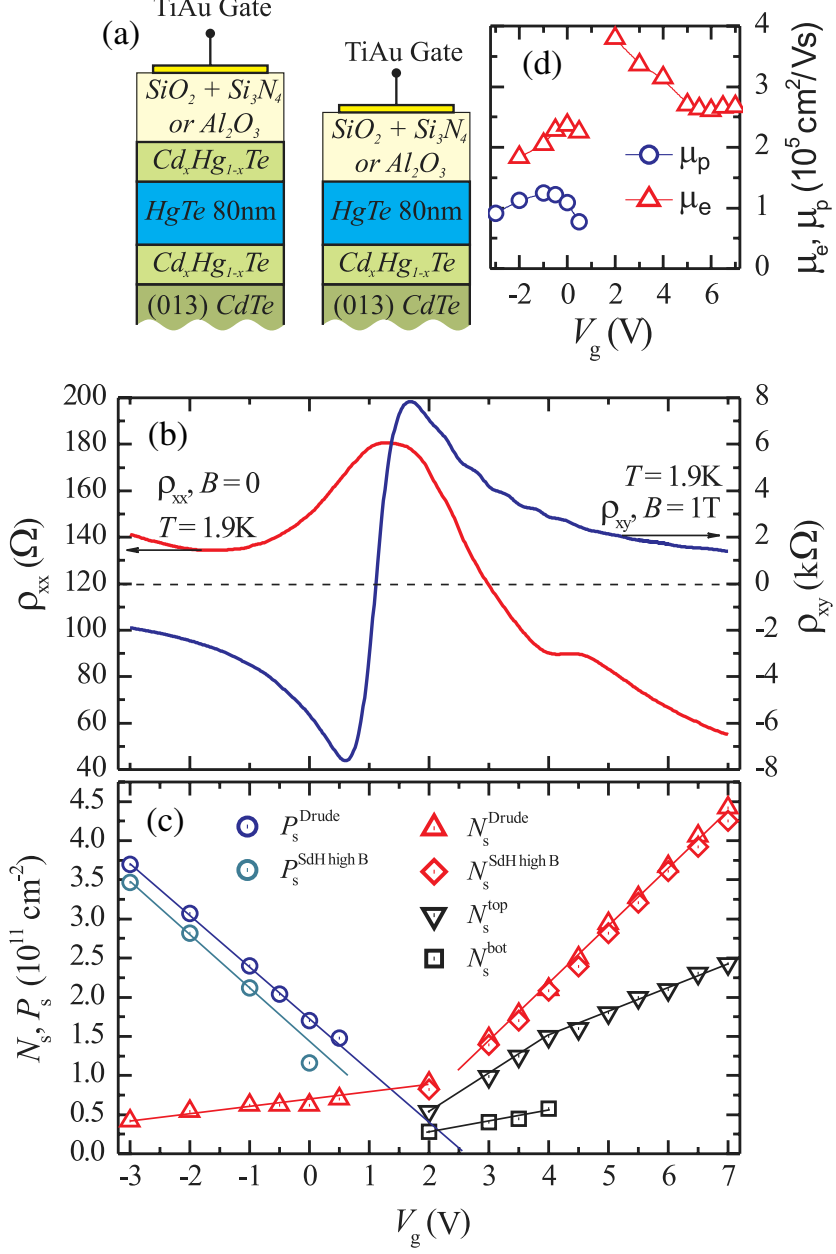

FIG. 1 (color online). (a) Cross section of our heterostructures. (b) Left vertical axis: $V_{g}$ dependence of $\rho_{x x}$ at $T=1.9 \mathrm{~K}$ and for $B=0$. Right vertical axis: Hall resistance $\left.\rho_{x y}\left(V_{g}\right)\right|_{B=1 \mathrm{~T}}$ for the second sample. (c) Electron $N_{s}\left(V_{g}\right)$ and hole $P_{s}\left(V_{g}\right)$ densities at different $V_{g}$ extracted from the Drude model and high-field $\mathrm{SdH}$ oscillations. The electron density of the top layer $N_{s}^{\text {top }}$ is extracted from low-field $\mathrm{SdH}$ oscillations (see text). (d) Electron and hole mobilities averaged over all participating charge carriers at different $V_{g}$. Between $V_{g}=0.5$ and $2 \mathrm{~V}$, the fits to the twocarrier Drude model were not reliable enough to extract electron and hole mobilities (see the Supplemental Material [18]).

suggests that $E_{F}$ can be tuned as a function of $V_{g}$ from the conduction band to the valence band.

For $V_{g}<1 \mathrm{~V}$, the Fermi level is in the valence band, where according to band structure calculations (See the Supplemental Material in Ref. [16]), holes and Dirac electrons coexist. The coexistence of two types of charge carriers is experimentally supported by a large positive magnetoresistance $\rho_{x x}(B)$ and by a nonlinear $\rho_{x y}(B)$, typical for electron-hole systems $[19,20]$. To estimate the mobility and density of the coexisting electron and holes, we used the Drude formalism for two types of carriers. Examples are given in the Supplemental Material [18]. A similar analysis to extract the densities and mobilities of the two carriers has been employed previously in semimetallic HgTe quantum wells [20]. At $V_{g} \geq 2 \mathrm{~V}$, the nonlinear Hall effect turns into an essentially linear dependence of $\rho_{x y}(B)$ indicating that the current is carried by only one sort of carrier, i.e., surface electrons. A remaining small nonlinearity of $\rho_{x y}(B)$ is due to different densities and mobilities of these electrons on the top and bottom surfaces.

The resulting electron and hole 2D density and mobility are presented in Figs. 1(c) (labeled as "Drude") and 1(d) [21]. Both the electron and hole densities change with $V_{g}$ by a factor of nearly 10 , thus, indicating that only a small concentration of bulk impurities contributes to the conductivity. Figure 1(d) shows that electrons and holes exhibit very high mobilities exceeding $10^{5} \mathrm{~cm}^{2} / \mathrm{V} \cdot \mathrm{s}$. The maximum electron mobility of $\mu=4 \times 10^{5} \mathrm{~cm}^{2} / \mathrm{V} \cdot \mathrm{s}$ is 10 times higher than in strained HgTe films discussed previously $[16,17]$ and almost 1,000 times higher than in 3D Bi TI samples. Linear extrapolation of the $P_{s}^{\text {Drude }}\left(V_{g}\right)$ data in Fig. 1(c) gives an intercept at $V_{g} \approx 2.5 \mathrm{~V}$, suggesting that the valence band filling starts around here. At about the same value of $V_{g}$, the slope of $N_{s}^{\text {Drude }}\left(V_{g}\right)$ in Fig. 1(c) and the temperature dependence of $\rho_{x x}\left(V_{g}\right)$ shown in Fig. 2(a) change noticeably: For $V_{g}>2 \mathrm{~V}, \rho_{x x}$ hardly changes with $T$ but changes dramatically for $V_{g}<2 \mathrm{~V}$ where $\rho_{x x}$ varies by a factor of 2 between $T=1.9$ and $15 \mathrm{~K}$. This behavior is ascribed to strong Landau scattering [22] of coexisting electron and hole states, similar to the one observed in Ref. [23].

Another feature in $\rho_{x x}\left(V_{g}\right)$ emerges at $V_{g}=4 \mathrm{~V}$ [Fig. 1(b)] accompanied by a change of the slope of $N_{s}^{\text {top }}\left(V_{g}\right)$ [Fig. 1(c)] extracted from low-field Shubnikov-de Haas (SdH) oscillations (see below). We suggest that these features mark the gate voltage at which $E_{F}$ starts to enter the conduction band. Therefore, the data presented in Figs. 1 and 2(a) imply that the gap opens between 2 and $2.5 \mathrm{~V}$ (top of valence band) and closes around $4 \mathrm{~V}$ (bottom of conduction band). Then, only Dirac states localized at the two surfaces of the strained $80 \mathrm{~nm}$ HgTe film contribute to transport. Outside this $V_{g}$ region, Dirac electrons and bulk electrons (holes) conduct in parallel. A sketch of the corresponding density of states (DOS) versus energy is shown in the inset of Fig. 2(a). Using the electron densities extracted at $E_{v}$ and $E_{c}$ and the calculated $k$-linear dispersion of Dirac electrons [16], we estimate a gap size of $\approx 15 \mathrm{meV}$. This value is very close to the one calculated in Ref. [16] for a strained HgTe film. The same value we found for the $\mathrm{Cd}_{0.7} \mathrm{Hg}_{0.3} \mathrm{Te}$ capped $\mathrm{HgTe}$ film, indicating that the surface states are not affected by the precise nature of the interface.

To check the validity of our picture further, we resort to magnetoresistance measurements in a perpendicular magnetic field displayed in Fig. 2(b). The magnetoresistance (MR) within classical Drude theory for two groups of carriers (labeled by indexes 1 and 2) is for small fields proportional to $B^{2}$. Its magnitude normalized to $\rho_{x x}(B=0)$ is proportional to $\left(\left(\sigma_{1} \sigma_{2}\right) /\left(\sigma_{1}+\sigma_{2}\right)^{2}\right)\left(\mu_{1} \mp \mu_{2}\right)^{2}$, where $\sigma_{i}$ 


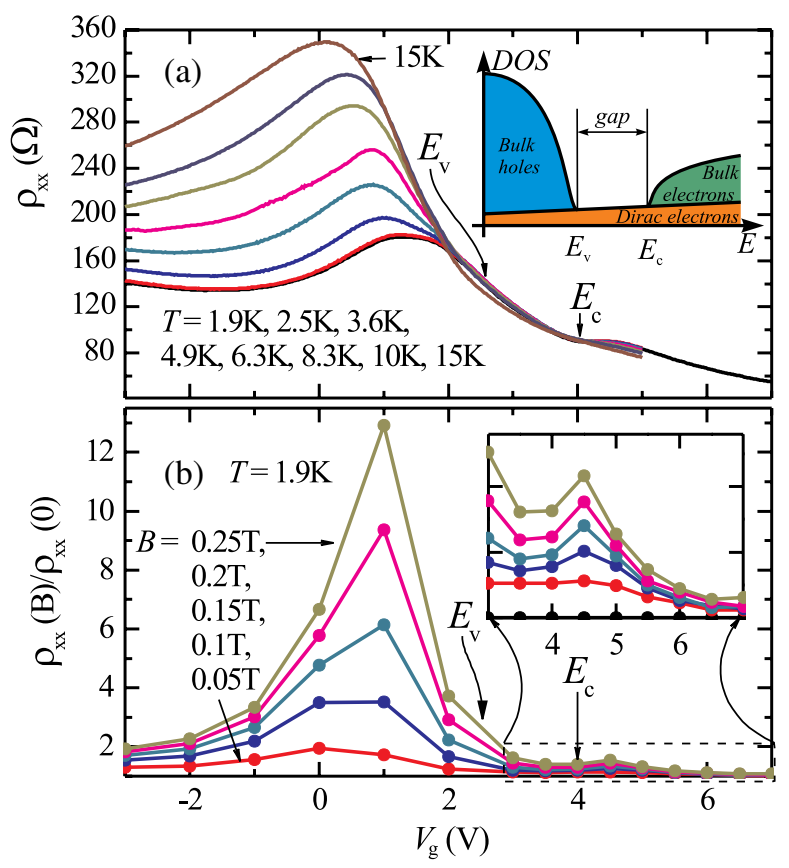

FIG. 2 (color online). (a) $\rho_{x x}\left(V_{g}\right)$ for different temperatures. The inset sketches the density of states in our system. (b) $\rho_{x x}(B) / \rho_{x x}(B=0)$ versus $V_{g}$ for different $B$. " $E_{v}$ " and " $E_{c}$ " mark the onset of the valence and conduction band.

and $\mu_{i}$ are the conductivity and mobility of the respective carrier species $i$ at $B=0$. The sign between $\mu_{i}$ depends on the carriers' polarity: in case they have the same polarity (e.g., Dirac electrons in the conduction band) the "-." sign applies, while in the case of different polarity (electrons and holes coexist), the mobilities add, thus, resulting in a strong MR. Hence, the maximum of the MR magnitude is expected to occur in the semimetal state with $E_{F}$ residing in the valence band near the charge neutrality point, where $N_{s} \approx P_{s}$ and $\sigma_{1}$ and $\sigma_{2}$ become comparable. A significant change of the MR magnitude is expected when $E_{F}$ moves from the valence band into the gap where only Dirac electrons reside. For this single carrier type, the MR is small. The corresponding normalized $\Delta \rho_{x x}\left(V_{g}\right)$ and $\Delta \rho_{x x}(B)$ data displayed in Fig. 2(b) are in accord with this expectation. For $V_{g}<2 \mathrm{~V}$, a large parabolic MR is observed with a MR maximum at $V_{g}=1 \mathrm{~V}$. In contrast, for $V_{g}>2 \mathrm{~V}$, i.e., where Dirac electrons prevail, the MR drops by a factor of up to 10 . The MR is expected to rise again when the Fermi level moves from the gap into the conduction band, as two (or more) groups of carriers with different mobility are involved, i.e., Dirac and bulk electrons. Bulk electrons near the bottom of the conduction band are expected to have a much lower mobility than Dirac electrons. A corresponding increase is, indeed, visible in Fig. 2(b) (marked by " $E_{c}$ ").

Important extra information can be obtained from experiments in quantizing $B$. SdH oscillations and quantized Hall steps can be seen in Fig. 3(a) as a function of $V_{g}$ for
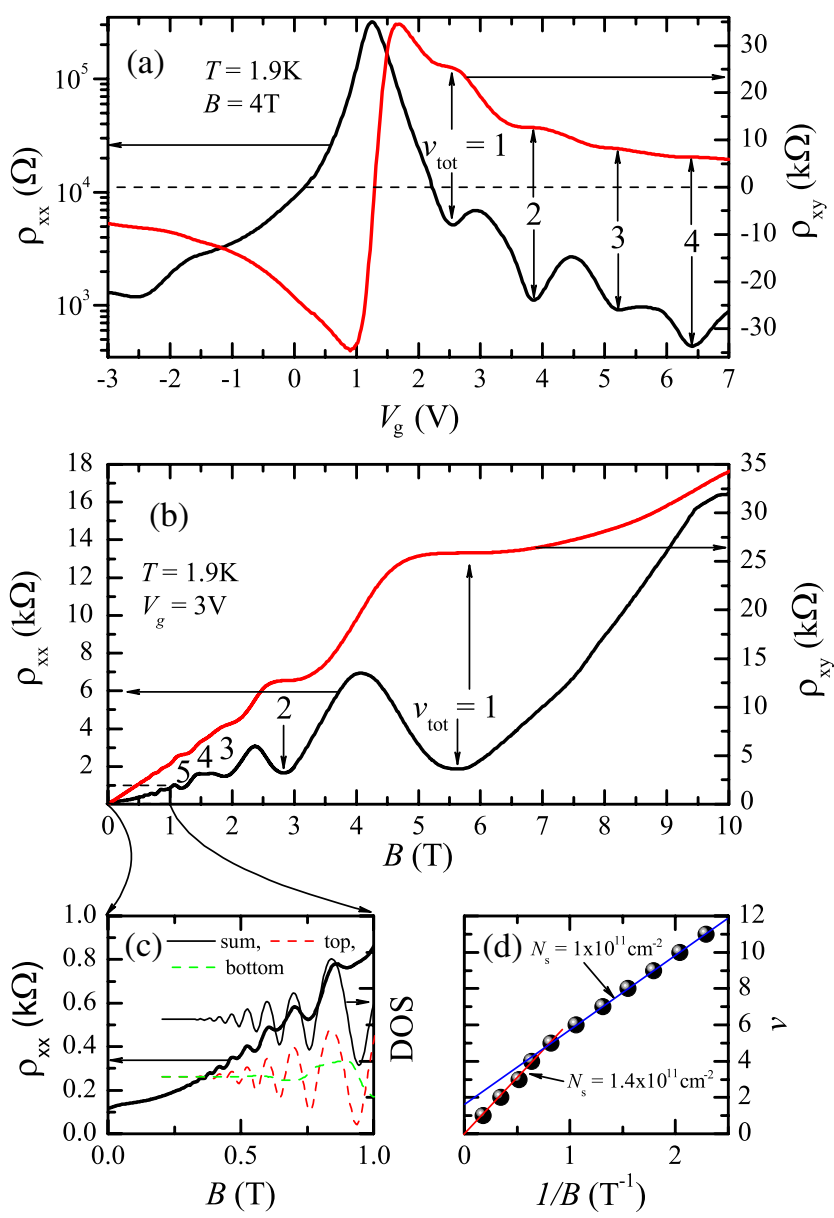

FIG. 3 (color online). (a) $V_{g}$ dependence of $\rho_{x x}$ and $\rho_{x y}$ at $4 \mathrm{~T}$. Numbered arrows mark the total filling factors $\nu^{\text {tot }}$. (b) Magnetoresistance $\rho_{x x}$ and Hall resistance $\rho_{x y}$ for $V_{g}=3 \mathrm{~V}$, i.e., in the TI regime. (c) Left axis: Magnification of low-field $\rho_{x x}(B)$. Right axis: calculated DOS for $N_{s}^{\text {top }}=1.015 \times 10^{11} \mathrm{~cm}^{-2}$ and $N_{s}^{\text {bot }}=$ $0.4 \times 10^{11} \mathrm{~cm}^{-2}$. The simulation follows closely the one described in Ref. [16]. Fitting $E_{F}(B=0)$, the energy shift between the fan chart for the top and bottom surface electrons $\Delta$ (see, also, the Supplemental Material in Ref. [16]) and the Landau level width (assuming a Gaussian) and keeping $N_{s}^{\text {top }}+N_{s}^{\text {bot }}$ constant, one can calculate the DOS of the different subsystems. The total low-field DOS is dominated by the top layer and describes the observed minima positions of $\rho_{x x}(B)$ correctly. (d) $\rho_{x x}(B)$ minima positions on a $1 / B$ scale with the corresponding densities.

$B=4 \mathrm{~T}$. The maximum in $\rho_{x x}$ at $V_{g} \sim 1 \mathrm{~V}$ corresponds to the charge neutrality point at which the Hall resistance changes sign. On the left, where low-mobility holes dominate, no quantum Hall steps appear. In contrast, on the right, where Dirac fermions and conduction band electrons prevail, quantized Hall steps develop.

Surprisingly, the Hall steps extend into a $V_{g}$ region where conduction band and Dirac electrons coexist. The electron density extracted from high-field $\mathrm{SdH}$ oscillations plotted in Fig. 1(c) is identical to the one obtained from classical Drude fits. This means that the filling factors at high $B$ fields are determined by the total electron 
concentration $N_{s}^{\text {tot }}$, i.e., bulk and surface electrons. Similarly, at large negative $V_{g}$ and high $B$, the hole density extracted from $\mathrm{SdH}$ oscillations and from Drude theory are nearly equal, while at smaller bias, the $\mathrm{SdH}$ data deliver smaller hole densities. This suggests that the filling factor in the valence band is given by the difference of bulk hole and surface electron density. This is similar to the situation observed in $\mathrm{GaSb} / \mathrm{InAs}$ heterojunctions where electrons and holes coexist [24]. A central observation is, thus, that bulk (which, in our case, is an $80 \mathrm{~nm}$ wide $\mathrm{HgTe}$ quantum well) and surface charge carriers determine jointly the high- $B$ Landau levels' (LLs) filling factors. The $\rho_{x x}(B)$ minima do not vanish, indicating parallel conduction probably due to the sides of the HgTe layer, which are oriented parallel to the applied $B$ [16]. The quantized Hall steps on the electron side display, as in Ref. [16], even and odd integer plateau values, thus, indicating different carrier densities for the top and bottom surfaces.

This is not surprising, as it is a consequence of screening; i.e., a part of the electric field gets screened by the top layer of Dirac electrons. Applying an electric field to the top gate, hence, results in different filling rates $d N_{s}^{\text {top(bot) }} / d V_{g}$. These rates can be easily estimated if $E_{F}$ is in the gap, i.e., between $\sim 2$ and $4 \mathrm{~V}$. Then the change of top and bottom electron density is given by [25] $\Delta N_{s}^{\text {top }} / \Delta N_{s}^{\text {bot }}=1+\left(q^{2} D d_{\mathrm{HgTe}} / \varepsilon_{\mathrm{HgTe}} \varepsilon_{0}\right)$, where $D$ is the DOS of Dirac electrons on the top surface, and $d_{\mathrm{HgTe}}$ and $\varepsilon_{\mathrm{HgTe}}$ are the thickness and dielectric constant of the $\mathrm{HgTe}$ layer, respectively. Inserting typical values, we obtain $\Delta N_{s}^{\text {top }} / \Delta N_{s}^{\text {bot }}=3-5$. Below we show that the experimentally observed difference in the top and bottom layer filling rate is close to this expected value. Besides $N_{s}^{\text {tot }}$, we plot in Fig. 1(c) also the electron density of the top surface as a function of $V_{g}$. The corresponding data are obtained from the following consideration: Assuming that the carrier densities are equal at the flatband condition, i.e., at $V_{g}=0 \mathrm{~V}$, the electron density on the top surface becomes significantly higher for $V_{g}>2 \mathrm{~V}$. The higher carrier density of the top surface is expected to be connected with a higher electron mobility [26]. This offers an opportunity to separate the electron density of the top and bottom layers experimentally. As the higher mobility of the top layer is connected to a smaller Landau level broadening, $\mathrm{SdH}$ oscillations commence at lower $B$ and dominate the low-field magnetoresistance oscillations in Fig. 3(c). This is reflected in different periods of $\mathrm{SdH}$ oscillations in the low and high $B$ fields displayed in Fig. 3(d). The $1 / B$ positions of the $\mathrm{SdH}$ minima versus filling factors can be fitted by two straight lines corresponding to the carrier density $N_{s}^{\text {top }}=1 \times 10^{11} \mathrm{~cm}^{-2}$ of the top layer and the total carrier density $N_{s}^{\text {tot }}=$ $1.4 \times 10^{11} \mathrm{~cm}^{-2} . N_{s}^{\text {top }}$ extracted from low-field SdH oscillations for different $V_{g}$ is shown in Fig. 1(c). In both cases, we assumed spin-resolved LLs. The reduced slope of
$N_{s}^{\text {top }}\left(V_{g}\right)$ for $V_{g}>4 \mathrm{~V}$ is a clear signature that $E_{F}$ moves in the conduction band. Since $d N_{s}^{\text {tot }} / d V_{g}$ is constant, $d N_{s}^{\text {top }} / d V_{g}$ decreases when the bulk electron density $N_{s}^{\text {bulk }}$ starts to appear, i.e., when $d N_{s}^{\text {bulk }} / d V_{g}>0$. For $B>1 \mathrm{~T}$, Landau quantization gets resolved in the lower mobility bottom layer, too, and, due to electron redistribution, the two surfaces (and for $V_{g}>4 \mathrm{~V}$ also the bulk electrons) act like a single 2DEG with density $N_{s}^{\text {tot }}$. The redistribution of electrons in the TI state is possible via contacts, ungated conducting regions, and via side facets of the HgTe film.

That the low-field $\mathrm{SdH}$ oscillations stem from a single surface of Dirac electrons is additionally supported by the phase of the quantum oscillations: For the high-field oscillations in Fig. $3(\mathrm{~d}),\left(1 / B_{\min }\right) / \Delta_{1 / B}=\nu^{\text {tot }}$ holds. The integer value indicates the absence of a phase shift, as expected for conventional $\mathrm{SdH}$ oscillations. Here, $B_{\min }$ is the $B$ position of the $\mathrm{SdH}$ minima, and $\Delta_{1 / B}$ is the period of the oscillations on a $1 / B$ scale. In the case of the lowfield oscillations, also plotted in Fig. 3(d), we obtain $\left(1 / B_{\min }\right) / \Delta_{1 / B}=$ integer +0.64 , i.e., quantum oscillations with a phase shift of $0.64 \pm 0.023$. This is close to the value of 0.5 expected for Dirac fermions on a single surface of a topological insulator (see, e.g., Ref. [27]).

With $N_{s}^{\text {tot }}$ acquired from high-field $\mathrm{SdH}$ oscillations and $N_{s}^{\text {top }}$, we can for $E_{F}$ between $2 V<V_{g}<4 \mathrm{~V}$, i.e., in the TI regime, calculate the carrier density of the bottom layer, $N_{s}^{\text {bot }}$. The corresponding data are also shown in Fig. 1(c). The slope of $N_{s}^{\text {top }}\left(V_{g}\right)$ is by a factor of $\sim 3$ higher than the one of $N_{s}^{\text {bot }}\left(V_{g}\right)$. This is in line with the effect of screening discussed above.

In summary, we have shown that an analysis of magnetotransport data in strained high-mobility HgTe layers brings out the different carrier types contributing to transport at different Fermi level positions. An analysis of high- and low-field quantum oscillations highlights the interplay of the different carrier types and allows us to probe the carrier density of the top and bottom layers in the TI regime separately.

The authors acknowledge the assistance of C. Linz in $\mathrm{Al}_{2} \mathrm{O}_{3}$ insulator and gate fabrication. This work was supported by the German Science Foundation (DFG) via Research Unit 1483 and SPP 1666, by the Linkage Grant of IB of BMBF at DLR, the Russian Foundation for Basic Research, the Ministry of Education and Science of the Russian Federation, and the Russian Academy of Sciences (Physics and Technology of Nanostructures Program).

*Corresponding author. dimko@isp.nsc.ru

[1] C. L. Kane and E. J. Mele, Phys. Rev. Lett. 95, 146802 (2005). 
[2] C. L. Kane and E. J. Mele, Phys. Rev. Lett. 95, 226801 (2005).

[3] B. A. Bernevig and S. -C. Zhang, Phys. Rev. Lett. 96, 106802 (2006).

[4] B. A. Bernevig, T. L. Hughes, and S.-C. Zhang, Science 314, 1757 (2006).

[5] L. Fu and C. L. Kane, Phys. Rev. B 76, 045302 (2007).

[6] L. Fu, C. L. Kane, and E. J. Mele, Phys. Rev. Lett. 98, 106803 (2007).

[7] D. Hsieh, D. Qian, L. Wray, Y. Xia, Y. S. Hor, R. J. Cava, and M. Z. Hasan, Nature (London) 452, 970 (2008).

[8] Y. Xia, D. Qian, D. Hsieh, L. Wray, A. Pal, H. Lin, A. Bansil, D. Grauer, Y. S. Hor, R. J. Cava, and M. Z. Hasan, Nat. Phys. 5, 398 (2009).

[9] M. Z. Hasan and C. L. Kane, Rev. Mod. Phys. 82, 3045 (2010).

[10] X. -L. Qi and S.-C. Zhang, Rev. Mod. Phys. 83, 1057 (2011).

[11] J. G. Checkelsky, Y. S. Hor, M.-H. Liu, D.-X. Qu, R. J. Cava, and N.P. Ong, Phys. Rev. Lett. 103, 246601 (2009).

[12] A. A. Taskin and Y. Ando, Phys. Rev. B 80, 085303 (2009).

[13] D. -X. Qu, Y. S. Hor, J. Xiong, R. J. Cava, and N. P. Ong, Science 329, 821 (2010).

[14] D. Kim, S. Cho, N. P. Butch, P. Syers, K. Kirshenbaum, S. Adam, J. Paglione, and M. S. Fuhrer, Nat. Phys. 8, 459 (2012).

[15] M. Konig, S. Wiedmann, C. Brune, A. Roth, H. Buhmann, L. W. Molenkamp, X.-L. Qi, and S.-C. Zhang, Science 318, 766 (2007).

[16] C. Brune, C. X. Liu, E. G. Novik, E. M. Hankiewicz, H. Buhmann, Y. L. Chen, X. L. Qi, Z.X. Shen, S. C. Zhang, and L.W. Molenkamp, Phys. Rev. Lett. 106, 126803 (2011).

[17] E. B. Olshanetsky, Z. D. Kvon, S. S. Kobylkin, D. A. Kozlov, N. N. Mikhailov, S. A. Dvoretskii, and J. C. Portal, JETP Lett. 93, 526 (2011).

[18] See Supplemental Material at http://link.aps.org/ supplemental/10.1103/PhysRevLett.112.196801 for details.

[19] F. J. Blatt, Physics of Electronic Conduction in Solids (McGraw-Hill, New York, 1968).

[20] Z. D. Kvon, E. B. Olshanetsky, D. A. Kozlov, N. N. Mikhailov, and S. A. Dvoretskii, JETP Lett. 87, 502 (2008).

[21] Note that the experiment delivers averages over both surfaces and, in certain voltage regions, also over part of the bulk. See [18] for details.

[22] V. F. Gantmakher and Y. B. Levinson, Carrier Scattering in Metals and Semiconductors (North-Holland Physics Publishing, Amsterdam, 1987).

[23] E. B. Olshanetsky, Z. D. Kvon, M. V. Entin, L. I. Magarill, N. N. Mikhailov, I. O. Parm, and S. A. Dvoretsky, JETP Lett. 89, 290 (2009).

[24] E. E. Mendez, L. Esaki, and L. L. Chang, Phys. Rev. Lett. 55, 2216 (1985).

[25] S. Luryi, Appl. Phys. Lett. 52, 501 (1988).

[26] Comparison of CdHgTe capped and uncapped heterostructures (see [18] for details) proves that they have the same average mobility for $E_{F}$ in the gap. We, thus, conclude that for the same carrier density, the mobilities on the top and bottom surfaces are approximately the same.

[27] A. A. Taskin and Y. Ando, Phys. Rev. B 84, 035301 (2011). 